\title{
Induction Versus Escalation in Multiple Sclerosis: A 10-Year Real World Study
}

\author{
Luca Prosperini ${ }^{1}$. Chiara Rosa Mancinelli ${ }^{2}$. Claudio Marcello Solaro ${ }^{3}$. Viviana Nociti ${ }^{4,5}$. Shalom Haggiag ${ }^{1}$. \\ Cinzia Cordioli ${ }^{2}$ - Laura De Giglio ${ }^{6,7}$ - Nicola De Rossi ${ }^{2}$ - Simonetta Galgani ${ }^{1}$ • Sarah Rasia ${ }^{2}$ • Serena Ruggieri ${ }^{1,7}$. \\ Carla Tortorella $^{1} \cdot$ Ruggero Capra $^{2} \cdot$ Massimiliano Mirabella $^{4,5} \cdot$ Claudio Gasperini $^{1}$
}

Published online: 31 March 2020

(C) The American Society for Experimental NeuroTherapeutics, Inc. 2020

\begin{abstract}
In this independent, multicenter, post-marketing study, we directly compare induction immunosuppression versus escalation strategies on the risk of reaching the disability milestone of Expanded Disability Status Scale (EDSS) $\geq 6.0$ over 10 years in previously untreated patients with relapsing-remitting multiple sclerosis. We collected data of patients who started interferon beta (escalation) versus mitoxantrone or cyclophosphamide (induction) as initial treatment. Main eligibility criteria included an EDSS score $\leq 4.0$ at treatment start and either $\geq 2$ relapses or 1 disabling relapse with evidence of $\geq 1$ gadolinium-enhancing lesion at magnetic resonance imaging scan in the pre-treatment year. Since patients were not randomized to treatment group, we performed a propensity score (PS)-based matching procedure to select individuals with homogeneous baseline characteristics. Comparisons were then conducted using Cox models stratified by matched pairs. Overall, 75 and 738 patients started with induction and escalation, respectively. Patients in the induction group were older and more disabled than those in the escalation group $(p<0.05)$. The PS-matching procedure retained 75 patients per group. In the re-sampled population, a lower proportion of patients reached the outcome after induction $(21 / 75,28.0 \%)$ than escalation $(29 / 75,38.7 \%)$ (hazard ratio $=0.48 ; p=0.024)$. Considering the whole sample, serious adverse events occurred more frequently after induction $(8 / 75,10.7 \%)$ than escalation (18/ $738,2.4 \%$ ) (odds ratio $=3.36, p=0.015$ ). These findings suggest that, in patients with poor prognostic factors, induction was more effective than escalation in reducing the risk of reaching the disability milestone, albeit with a worse safety profile. Future studies are warranted to explore if newer induction agents may provide a more advantageous long-lasting risk:benefit profile.
\end{abstract}

Electronic supplementary material The online version of this article (https://doi.org/10.1007/s13311-020-00847-0) contains supplementary material, which is available to authorized users.

Luca Prosperini

luca.prosperini@gmail.com

Chiara Rosa Mancinelli

mancinellichiara86@gmail.com

Claudio Marcello Solaro

csolaro.centrosm@gmail.com

Viviana Nociti

viviana.nociti@policlinicogemelli.it

Shalom Haggiag

lvshalom@hotmail.com

Cinzia Cordioli

cinzia.cordioli@gmail.com

Laura De Giglio

laura.degiglio@gmail.com

Nicola De Rossi

ncl.derossi@gmail.com

\author{
Simonetta Galgani \\ sgalgani@scamilloforlanini.rm.it \\ Sarah Rasia \\ sarah.rasia@gmail.com \\ Serena Ruggieri \\ serena.ruggieri@gmail.com \\ Carla Tortorella \\ carla.tortorella@gmail.com \\ Ruggero Capra \\ ruggero.capra@gmail.com \\ Massimiliano Mirabella \\ m.mirabella@icloud.com \\ Claudio Gasperini \\ c.gasperini@libero.it
}

Extended author information available on the last page of the article 
Key Words Multiple sclerosis $\cdot$ induction $\cdot$ escalation $\cdot$ therapeutic algorithm

\section{Introduction}

Two main treatment strategies are currently recognized to prevent clinical relapses, disability accumulation, and inflammatory activity on magnetic resonance imaging (MRI) in patients with relapsing-remitting multiple sclerosis (RRMS): escalation and early intensive treatment [1].

Escalation consists of starting with less potent and safest immunomodulatory drugs and gradually switch to highefficacy treatments in case of breakthrough disease.

The rationale behind escalation strategy is that patients at the earlier disease stage may respond optimally to a safer, lowefficacy immunomodulatory injectable or oral treatments [2], such as interferon beta (IFNB), glatiramer acetate (GA), teriflunomide (TFN), and dimethyl fumarate (DMF). If disease activity persists despite therapy, escalation to high-efficacy treatments with non-selective intravenous (iv) immunosuppressant agents (mitoxantrone (MTX), cyclophosphamide (CYC)), or with selective immunosuppression by either oral drugs (fingolimod (FNG), cladribine (CLB)) or monoclonal antibodies (natalizumab (NTZ), alemtuzumab (ALZ), ocrelizumab (OCR), rituximab (RTX)), is warranted to avoid further relapses and future disability. However, this approach requires careful evaluation of treatment response, an issue lacking of consensus on definition and monitoring actions [3]. In addition, the escalation approach may potentially expose patients to miss the socalled therapeutic window, i.e., postponing high-efficacy treatments until when neurodegeneration prevails over inflammation $[4,5]$.

Early (i.e. shortly after the diagnosis) intensive treatment includes durable therapy with high-efficacy drugs (NTZ, FNG, OCR, RTX) and induction obtained with potent immunosuppressant drugs, followed by a maintenance immunomodulating therapy. This latter approach requires short-time administration of immunosuppressant agents whose biological effect are longlasting and not quickly reversible following treatment discontinuation [6], such as CYC, MTX, ALZ, and CLB. Therefore, though NTZ, OCR, RTX, and, to some extent, even FNG are considered high-efficacy treatments, they are not suitable as induction agents $[7,8]$.

The rationale behind induction strategy relies on "resetting" the immune system in order to achieve an early disease control [7, 8]. Exposure to induction immunosuppression should ideally last as short as possible, to minimize the risk of malignant neoplasms $[9,10]$ and opportunistic infections [11].

Currently in clinical practice, escalation is intended to be suitable for most patients [1], while induction is mainly restricted to patients with aggressive RRMS [12]. However, the escalation approach has recently been criticized since considered not adequate or not sufficient in conferring the greatest possible long-lasting therapeutic effect [13]. Because randomized clinical trial addressing escalation versus induction in MS are lacking, further contributing to uncertainty on how start treatment $[5,14]$, observational data with quasi-experimental design might help to fill this gap. Therefore, here we evaluated the long-term (10 years) effectiveness of initial treatment with escalation versus induction approach in RRMS patients, using a multicenter, retrospective local MS registry data.

\section{Methods}

\section{Study Design}

This was an independent, multicenter, post-marketing study.

We retrospectively analyzed data of patients affected by RRMS who regularly attended 5 tertiary MS outpatient clinics in Italy: S. Camillo-Forlanini Hospital, Rome; S. Andrea Hospital, Rome; Policlinico “A. Gemelli," Rome; ASST Spedali Civili di Brescia, Montichiari (BS); Rehabilitation Unit 'Mons. L. Novarese' Hospital, Moncrivello (VC).

Clinical data were prospectively collected by each MS center following the local medication monitoring plan and hospital guidelines and then stored in an ad hoc electronic database developed for this study. In no way this study did interfere with the usual care and monitoring received by patients.

\section{Participants}

We collected data of previously untreated patients starting a disease-modifying therapy with either an immunomodulatory agent (IFNB or GA), possibly switched to high-effective treatments (MTX, CYC, NTZ, ALZ, FNG, or CLB) in case of treatment failure (escalation group), or with iv immunosuppression (MTX or CYC), followed or not by maintenance treatments (induction group).

The inclusion criteria at treatment start (henceforth defined as "baseline") were as follows:

(1) Age $<55$ years

(2) RR disease course [15]

(3) Treatment-naïve status

(4) $<5$ years since the first demyelinating event, the time frame in which disease activity is most correlated with long-term disability [16, 17], and there is the greater risk reduction of transition to secondary progressive MS by treatment [18]; 
(5) An Expanded Disability Status Scale (EDSS) score $\leq 4.0$ [19], indicating a threshold where the disability accumulation is mainly driven by inflammation rather by neurodegeneration [20]

(6) Available brain MRI scan performed within 1 month before initial treatment with escalation or induction strategy (baseline MRI scan)

(7) "Active" disease, defined as either $\geq 2$ relapses in the pretreatment year or 1 relapse with residual disability and $\geq 1$ gadolinium-enhancing lesion at baseline MRI scan (this definition of 'active' disease was adapted from a previous observational study exploring the effectiveness and safety of MTX as induction strategy in "aggressive" RRMS [21])

(8) At least two clinical evaluations per year including disability scoring with EDSS performed by certified neurologists (www.neurostatus.net)

The exclusion criteria at baseline were as follows:

(1) Primary or secondary progressive MS [15]

(2) Patients lost to follow-up before 10 years of observation for reasons other than death

\section{Outcome Definition}

Main Outcome Time to reach the disability milestones of EDSS score $\geq 6.0$, corresponding to the ability to walk only with unilateral support and $<100 \mathrm{~m}$ without resting, confirmed in at least two consecutive visits and sustained (stable or higher) over the entire follow-up [19]. We adopted such outcome instead of the classical 0.5 or 1.0-point EDSS worsening [22] to set a robust endpoint based on a clinically significant milestone for patients with MS.

Secondary Outcome EDSS score assessed 10 years after the treatment start.

Additional Outcome Serious adverse events (SAEs) defined as any untoward medical occurrence that at any dose resulted in death, was life-threatening, required inpatient hospitalization or causes prolongation of existing hospitalization, resulted in persistent or significant disability/incapacity, might have caused a congenital anomaly/birth defect, or required intervention to prevent permanent impairment or damage (http:// ichgcp.net/12-adverse-event-ae).

\section{Statistical Analysis}

Categorical data were presented as count (proportion); continuous data were presented as mean \pm standard deviation (SD) or median (interquartile range), unless indicated otherwise.
We collected the following baseline variables: sex, age, disease duration (i.e., the time elapsed since symptom onset), EDSS score, number of relapses in prior year, absence or presence of gadolinium-enhancing lesions on brain MRI scan.

Differences in baseline characteristics between escalation and induction groups were tested with the Fisher exact test or the Mann-Whitney $U$ test for categorical or continuous variables, as appropriate.

As patients were not randomized to treatment group (induction or escalation), we performed a $1: 1$ ratio matching procedure combining an exact matching on sex with a propensity score (PS)-based nearest-neighbor matching within a caliper of 0.05 (without replacement). Individual PS values were estimated by use of logistic regression with the aforementioned baseline characteristics as covariates (sex, age, disease duration, EDSS score, number of relapses in prior year, absence or presence of gadolinium-enhancing lesions), and treatment group as the dependent variable. The validity of PS-based matching was tested by analysis of standardized differences $(|d|)$, with $|d|>0.20$ considered as imbalance [23].

We compared escalation and induction on the risk of reaching EDSS score $\geq 6.0$ (primary outcome) using a Cox regression model stratified by matched pairs [24]. The time elapsed from baseline to the last visit over the 10-year follow-up or outcome reach (whichever came first) was entered as main time variable in the models. This procedure allowed us to exclusively select patients with similar baseline characteristics and to obtain a comparable follow-up length for each pair [25]. Graphic inspection of log-minus-log survival plots confirmed the proportional hazard assumption in post-matching Cox analysis.

To assess robustness of the results, we also conducted several post-estimation sensitivity analyses as follows:

(1) After inserting in the Cox model the time since first symptom to EDSS score $\geq 6.0$ (rather than the time since baseline) as main time variable

(2) After entering in the Cox model not only the treatment strategy, but also all the baseline variables

(3) After entering the time (years) on high-effective treatments as time-varying covariate

(4) After re-running the Cox model with the "best" $n: 1$ matching procedure among ratios of $2: 1,3: 1$, and $4: 1$ to provide more precise estimations (through larger sample size) without compromising the balance across covariates [26]

Between-group comparison of the last EDSS score at 10 years (secondary outcome) was carried out in matched pairs by the Wilcoxon signed-rank test. 
Lastly, in the whole sample, we compared the proportions of patients with SAEs in the two groups with a logistic regression analysis adjusted for baseline variables.

Two-tailed $p$ values $<0.05$ were considered significant.

\section{Results}

\section{Participants}

We examined records from 3851 patients in the escalation group and 132 in the induction group who started treatment from 1998 to 2009 (see Fig. 1 for the study flowchart).

Within the escalation group, 738 out of 3851 were included in the analysis. Excluded patients had longer disease duration and fewer pre-treatment relapses and were less likely to have gadolinium enhancement at the baseline MRI scan ( $p$ values $<0.05$ ).

Within the induction group, 75 out of 132 patients were included in the analysis. Excluded patients had longer disease duration and higher EDSS score at baseline ( $p$ values $<0.05$ ).

\section{Findings in the Whole Cohort}

All patients in the escalation group $(n=738)$ started with highdose, high-frequency IFNB-1a or IFNB-1b; of them, 394 $(53.4 \%)$ required one or more high-efficacy treatments after a median time of 3.5 ( 2 to 5.5$)$ years, namely NTZ $(n=234)$,
FNG $(n=74), \operatorname{MTX}(n=62), \operatorname{ALZ}(n=8)$, CYC $(n=7)$, CLB $(n=5)$, and rituximab $(n=4)$. The remaining $344(46.6 \%)$ continued to receive the same initial treatment $(n=190)$ or switched to different low-efficacy treatments after a median time of 6 (3 to 6.5) years such as DMF $(n=76)$, GA $(n=37)$, TFN $(n=26)$, or azathioprine $(n=15)$ over the 10-year follow-up (Fig. 2).

Patients in the induction group $(n=75)$ started with monthly or bi-monthly infusions for 6-24 months of either MTX $(n=55)$ at the dosage of $8 \mathrm{mg} / \mathrm{mq}$ (maximum cumulative dose, $140 \mathrm{mg} / \mathrm{mq})$ or CYC $(n=20)$ at the dosage of 500 to $1000 \mathrm{mg} / \mathrm{mq}$. They received a median (range) number of 8 (6-12) MTX infusions and 10 (6-12) CYC infusions, respectively. When necessary, dosage adjustment was done on the basis of blood cell count to avoid myelotoxicity and to ensure the expected level of immunosuppression. Immediate postinduction MRI data were available only for 45 patients. All available MRI scans showed suppression of inflammatory activity (absence of gadolinium-enhancing lesions).

Immediately after the last immunosuppression infusion, 42 (56.0\%) patients received a maintenance treatment with IFNB $(n=20)$, GA $(n=14)$, or AZA $(n=8)$. Twenty-four $(32.0 \%)$ patients started IFNB $(n=22)$ or AZA $(n=2)$ because of the occurrence of relapses $(n=16)$ or isolated MRI activity $(n=8)$ after a median time of 1.25 ( 1 to 2 ) years from the last immunosuppression infusion. The remaining $9(12.0 \%)$ patients did not receive any treatment following induction immunosuppression since they were free from disease activity and did
Fig. 1 Study flowchart of patients' disposition
ESCALATION

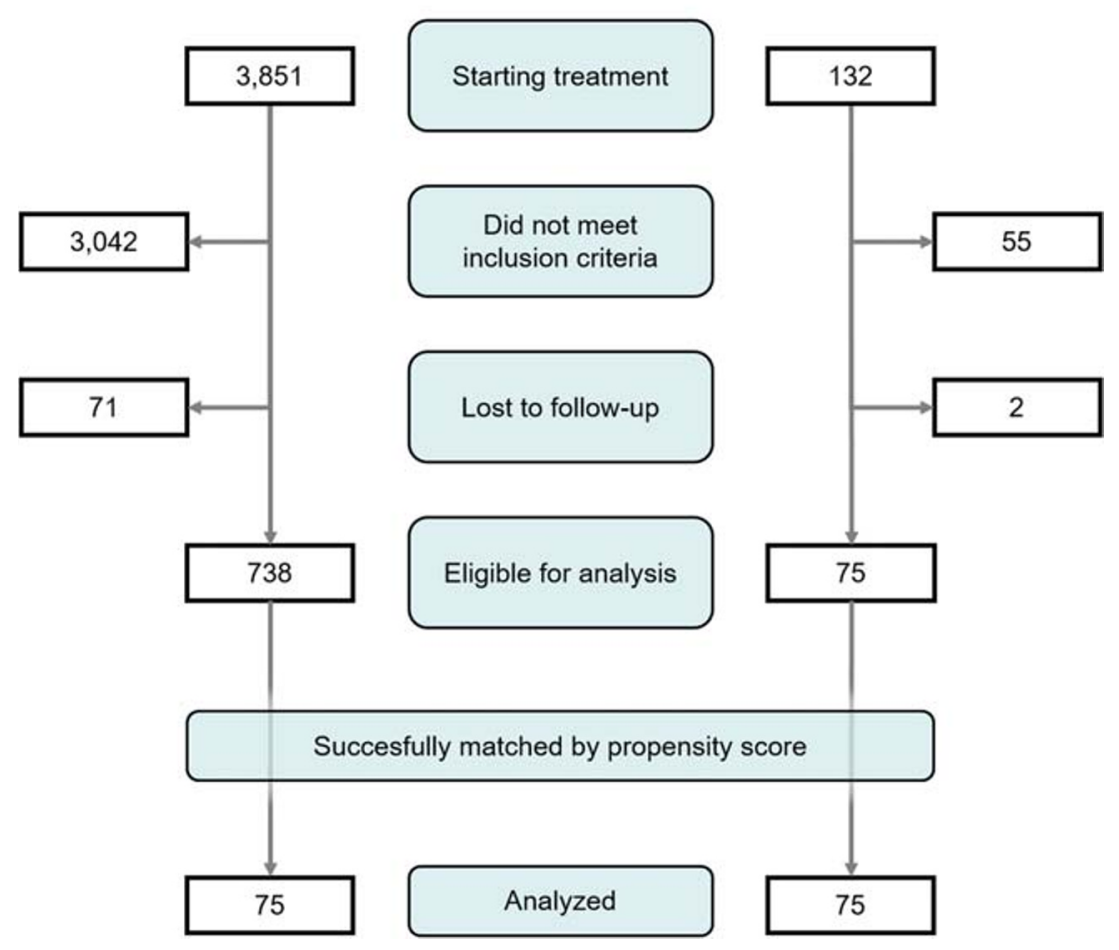



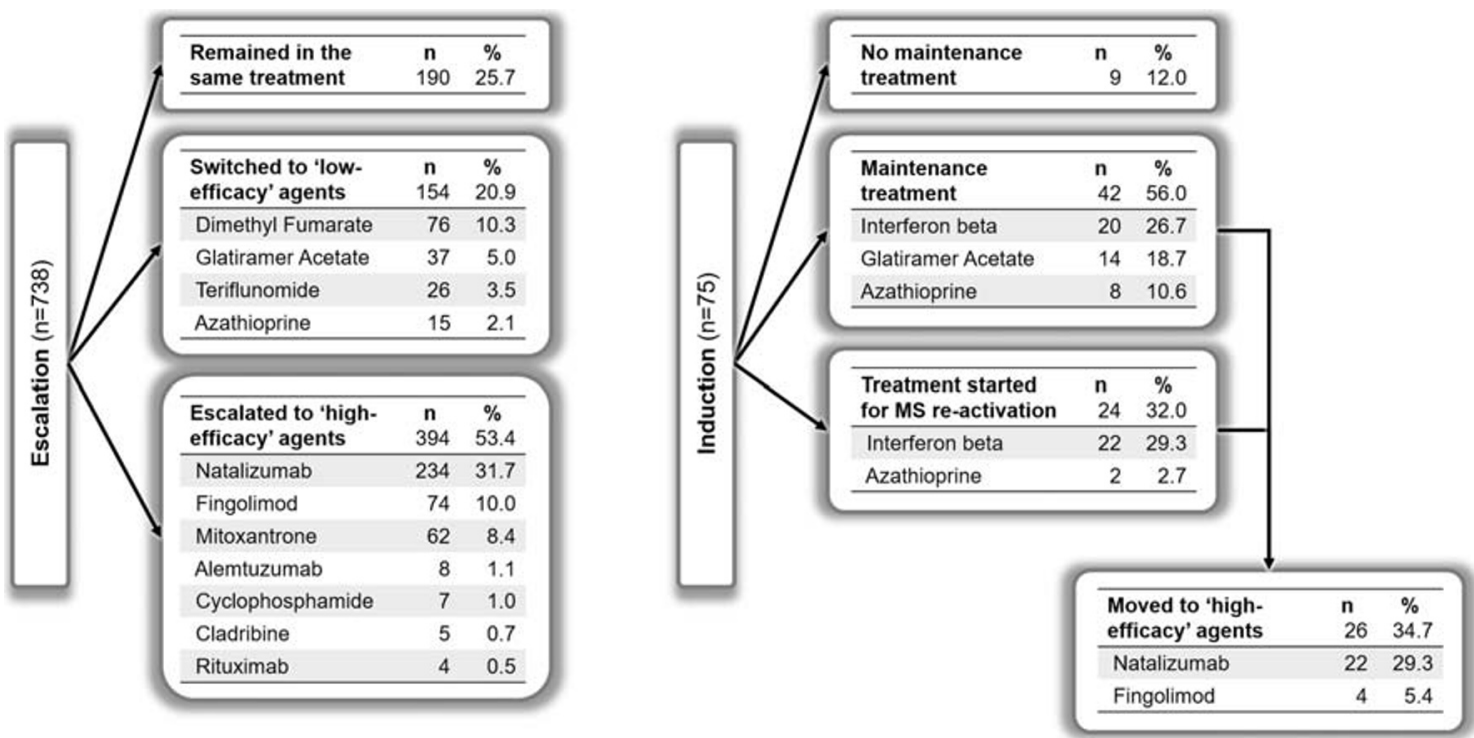

Fig. 2 Treatment sequencing after escalation and induction at 10-year follow-up (note that one patient in either group was submitted to autologous hematopoietic stem cell transplantation after high-efficacy treatments)

not accumulate disability over the 10 follow-up period (see also Fig. 2).

Despite induction, 26 (34.7\%) patients required more efficacious treatments, such as NTZ $(n=22)$ or FNG $(n=4)$, over the follow-up period. One patient in either group was submitted to autologous stem cell transplant after failing multiple high-efficacy treatments.

Considering the whole unmatched sample, 111/738 $(15.0 \%)$ and $21 / 75(28.0 \%)$ patients reached the outcome at follow-up in the escalation and induction groups, respectively $(p=0.008)$. The median final EDSS scores were 2.5 (1.5 to $4.5)$ and 4.5 (3.5 to 6.0) in the escalation and induction groups, respectively $(p<0.001)$.

\section{PS-Based Matching Procedure}

Table 1 shows the baseline variables of patients eligible for analysis in the unmatched cohort and after matching in a 1:1 ratio. We observed a significant imbalance in pre-matching baseline characteristics across treatment groups due to the older age $(p=0.002)$ and higher EDSS score $(p<0.001)$ in the induction group. This between-group imbalance did not persist after the matching procedures that retained 150 patients (75 per group). No covariate exhibited large imbalance $(|d|<$ 0.20 ) after the re-sampling procedures, and the standardized mean difference of PS values decreased by approximately $99 \%$, from 0.999 to 0.006 , indicating a significant improvement in the overall match.

\section{Follow-up Data in the Re-matched Samples}

After matching in a 1:1 ratio, we found that the proportion of patients reaching the primary outcome was lower in the induction $(n=21,28.0 \%)$ than in the escalation group $(n=$ 29, 38.7\%) (hazard ratio $[\mathrm{HR}]=0.48, p=0.024$ ) (Fig. 3A). Findings from the sensitivity analyses, providing results consistent with the core analysis, are shown in Table 2 . When the disease duration was set as main time variable instead of time from baseline, we observed that patients in the escalation group were more prone to approach the disability accrual of natural MS history than those in the induction group (Fig. 3B).

At 10-year follow-up, the median final EDSS scores in the $1: 1$ ratio re-sampled cohort were $5.0(3.5-6.5)$ and $4.5(3.5-$ 6.0) after escalation and induction, respectively $(Z=1.75, p=$ $0.08)$. We found consistent results even after matching in a $1: 2$ ratio $(Z=2.04, p=0.042)$.

\section{Baseline Variables Associated with Worse Outcome}

We explored if there were associations between the risk of reaching the main outcome and baseline variables other than treatment strategy (Table 3). As expected, the baseline EDSS score was associated with an increased risk of reaching the outcome after both escalation (HR $=3.15, p<0.001)$ and induction $(\mathrm{HR}=5.10, p<0.001)$. Within the escalation group, a longer disease duration at treatment start was also associated with an increased risk of reaching the outcome ( $\mathrm{HR}=1.38$ for each year of delay, $p=0.014$ ). Within the induction group, the only other factor associated with increased risk was an older age at treatment start (HR $=1.08$ for each year, $p=0.007)$. We found no association between the 10-year primary outcome and the type of induction agent (CYC versus MTX), the number of iv infusions of CYC or MTX, or the maintenance therapy following the induction immunosuppression ( $p$ values $>$ $0.2)$. 
Table 1 Baseline characteristics of the included patients before and after the matching procedure

\begin{tabular}{|c|c|c|c|c|c|c|}
\hline & \multicolumn{3}{|c|}{ Unmatched cohort } & \multicolumn{3}{|c|}{ Matched cohort (1:1 ratio) } \\
\hline & Escalation & Induction & $|d|$ & Escalation & Induction & $|d|$ \\
\hline$N$ & 738 & 75 & N/A & 75 & 75 & $\mathrm{~N} / \mathrm{A}$ \\
\hline Male sex, $n(\%)$ & $212(28.7)$ & $24(32.0)$ & N/A & $24(32.0)$ & $24(32.0)$ & $\mathrm{N} / \mathrm{A}$ \\
\hline Age, years & $32.2(8.4)$ & $35.6(9.3)^{*}$ & 0.37 & $36.3(8.8)$ & $35.6(9.3)$ & 0.04 \\
\hline Disease duration, years & $2.0(1.4)$ & $2.2(1.6)$ & 0.16 & $2.2(1.4)$ & $2.2(1.6)$ & 0.09 \\
\hline EDSS score, median [interval] & $1.5[0-4.0]$ & $2.5[1.0-4.0]^{*}$ & 1.69 & $2.5[1.0-4.0]$ & $2.5[1.0-4.0]$ & 0.01 \\
\hline No. of relapses in previous year & $2.1(0.8)$ & $2.2(0.8)$ & 0.13 & $2.2(0.9)$ & $2.2(0.8)$ & 0.12 \\
\hline Presence of GD-enhancement, $n(\%)$ & $460(62.3)$ & $43(58.1)$ & $\mathrm{N} / \mathrm{A}$ & $43(58.1)$ & $43(58.1)$ & $\mathrm{N} / \mathrm{A}$ \\
\hline Propensity score & $0.079(0.101)$ & $0.215(0.136)^{*}$ & 0.99 & $0.213(0.137)$ & $0.215(0.136)$ & 0.01 \\
\hline
\end{tabular}

All values are reported as mean (standard deviation) unless indicated otherwise

$* p<0.01$

$|d|$ refers to standardized difference (Cohen's $d$ )

N/A: not applicable

\section{Safety Data}

Considering the whole umatched population, SAEs occurred more frequently after induction $(n=8,10.7 \%)$ than escalation $(n=18,2.4 \%)$ : crude odds ratio $(\mathrm{OR})=4.78$ (95\% CIs 2.00 to $11.39 ; p=0.001)$, adjusted $\mathrm{OR}=3.36(95 \%$ CIs 1.26 to 8.95 , $p=0.015)$. We found no difference between MTX and CYC in terms of SAE occurrence $(p=0.98)$. After causality assessment, the treatment associated with the SAE occurrence was permanently discontinued in 11 patients, including 3 cases of NTZ-related progressive multifocal leukoencephalopathy; in 5 cases, the SAE occurred off-treatment after MTX (leukemia, $n=3$; breast cancer, $n=1$ ) or ALZ (Grave's disease, $n=1$ ) administration; 4 cases of solid malignancies occurred while

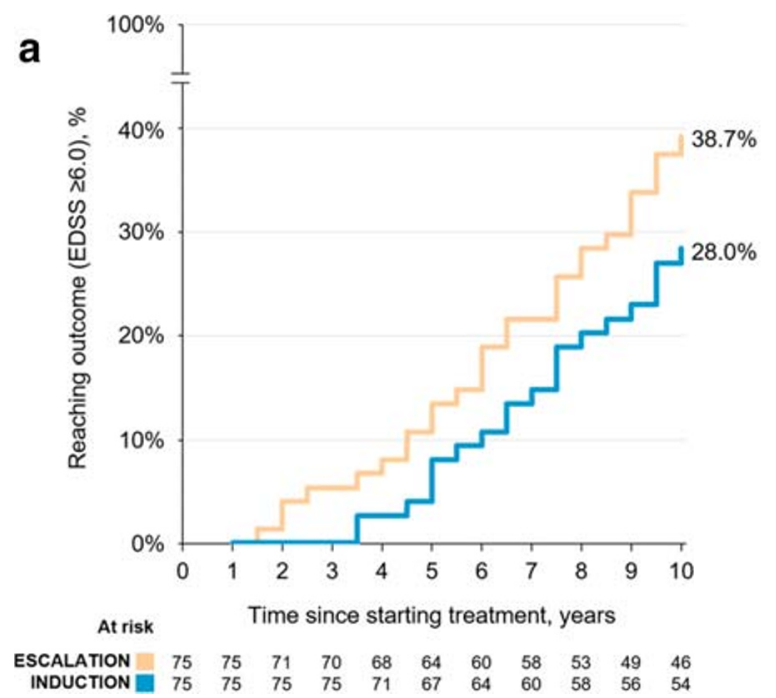

Fig. 3 Time since treatment start (A) and since symptom onset (B) to EDSS score $\geq 6.0$ by initial treatment strategy. The gray area overlaid in part B indicates the estimated median time since symptom onset to EDSS on IFNB $(n=3)$ or NTZ $(n=1)$, but they were probably related with previous exposure to MTX or CYC (see Table 4 for details).

\section{Discussion}

In this independent, multicenter, retrospective study, we conducted a PS-based matched analysis to explore the long-term effects of an initial treatment approach with escalation or induction in patients with RRMS. As per inclusion criteria and also through the PS-based matching procedure, we selected a sample of patients with evidence of "active" disease (the median pre-treatment annualized relapse rate was 2 and

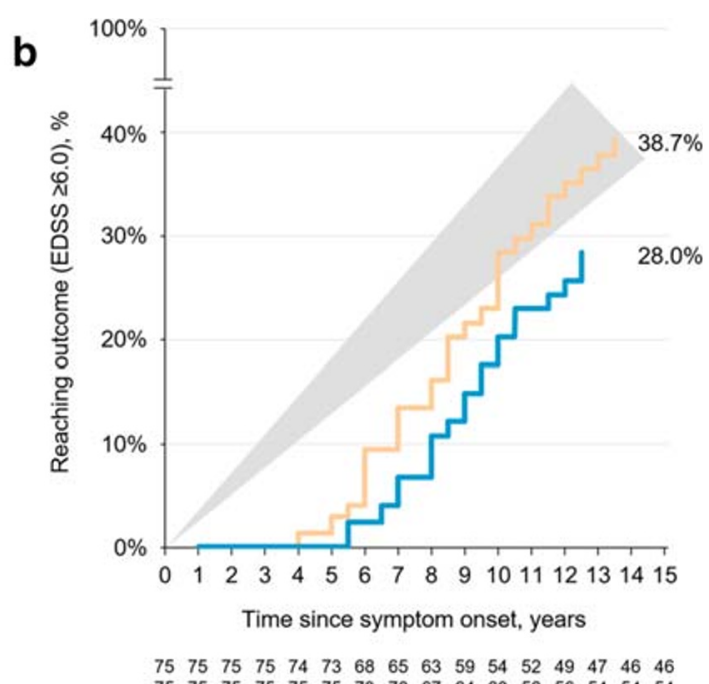

score $\geq 6.0$ in natural history studies ranging from 14 to 20 years (adapted from Confavreux and Vukusic [27]) 
Table 2 Time to EDSS score $\geq 6.0$ by initial treatment strategy (hazard ratios $<1.0$ indicate more favorable outcome for induction)

\begin{tabular}{|c|c|c|c|c|}
\hline & \multirow[t]{2}{*}{ Hazard ratio } & \multicolumn{2}{|c|}{$95 \%$ confidence intervals $*$} & \multirow[t]{2}{*}{$p$ value } \\
\hline & & Lower bound & Upper bound & \\
\hline Case base scenario & 0.48 & 0.18 & 0.97 & 0.024 \\
\hline \multicolumn{5}{|l|}{ Sensitivity analyses } \\
\hline (1) Model with time since first symptom as main time variable & 0.54 & 0.15 & 0.96 & 0.037 \\
\hline (2) Model adjusted by all baseline variables & 0.38 & 0.20 & 0.88 & 0.018 \\
\hline (3) Model adjusted by time on high-effective treatments & 0.47 & 0.14 & 0.92 & 0.027 \\
\hline (4) Model built after matching in a 2:1 ratio & 0.58 & 0.34 & 0.98 & 0.042 \\
\hline
\end{tabular}

*Based on 1000 bootstrap samples (bias corrected and accelerated)

approximately $60 \%$ of them had an active MRI scan at baseline) and poor prognostic factors (the median EDSS score at baseline was 2.5) in spite of a relatively short disease duration (2 years on average).

In our cohort, induction was associated with an approximately $50 \%$ reduced risk of reaching EDSS $\geq 6.0$ as compared to escalation, albeit with a worse safety profile. Notably, the probability of reaching EDSS $\geq 6.0$ in the escalation group overlaps, especially after 10 years from disease onset, with the estimated median disease duration since symptom onset to EDSS score $\geq 6.0$ reported in natural history studies, ranging from 14 to 20 years (see also Fig. 3B) [27]. The analysis of baseline variables associated with worse outcome also confirms that (1) the longer the time to treatment start, the worse the long-term outcomes when starting with escalation [18], and (2) younger age is associated with better outcome after induction [21].

The growing availability of therapeutic agents for RRMS has prompted renewed interest in the issue of treatment algorithm, but at the same time it is a matter of concern because of the unknown long-term immunologic and safety risks of sequencing multiple therapies [28]. While escalation is the more widely adopted treatment strategy, induction is restricted to patients at risk of rapid disability accrual, mainly because the poor definition of the target population and the increased risk of immunosuppression-related toxicity [2]. Although an induction approach is well established in hematologic malignancies [29] and in other autoimmune diseases (e.g., rheumatoid arthritis) [30], data supporting initial treatment with induction agents in RRMS are limited to few small clinical trials on MTX [31, 32], one observational study on CYC [33], and larger clinical trials on ALZ [34, 35] and CLB [36]. These studies have provided considerable results on relapse rate, time to sustained disability worsening, and MRI measures in the short-term period [31-36]. Our results are also in line with two real-world retrospective studies showing early intensive treatments (including NTZ and ALZ) as associated with reduced risk of transition to secondary progressive MS [18] and disability accumulation [13] than low-efficacy drugs.

Our study has the merit of a longer follow-up period (10 years) and an exclusive inclusion of patients initially treated with immunosuppressive agents suitable for induction. However, in our sample, a certain proportion of patients required high-efficacy treatments with monoclonal antibodies or FNG even after induction. Although this proportion was lower after induction than after escalation (34.7\% versus $53.4 \%$ ),

Table 3 Association between the risk of reaching the outcome of EDSS score $\geq 6.0$ at 10 years and baseline variables

\begin{tabular}{|c|c|c|c|c|c|c|}
\hline & \multicolumn{3}{|l|}{ Escalation } & \multicolumn{3}{|l|}{ Induction } \\
\hline & \multirow[t]{2}{*}{ Hazard ratio } & \multicolumn{2}{|c|}{$95 \%$ confidence intervals $*$} & \multirow[t]{2}{*}{ Hazard ratio } & \multicolumn{2}{|c|}{$95 \%$ confidence intervals $*$} \\
\hline & & Lower bound & Upper bound & & Lower bound & Upper bound \\
\hline Male sex & 0.78 & 0.32 & 1.89 & 0.60 & 0.19 & 1.93 \\
\hline Age (each year) & 1.04 & 0.98 & 1.09 & $1.08^{\#}$ & 1.03 & 1.15 \\
\hline Disease duration (each year) & $1.38^{\#}$ & 1.03 & 1.86 & 1.08 & 0.80 & 1.45 \\
\hline EDSS score (each step) & $3.15^{\#}$ & 1.68 & 5.91 & $5.10^{\#}$ & 2.01 & 12.95 \\
\hline No. of relapses in previous year & 1.24 & 0.75 & 2.27 & 1.27 & 0.67 & 2.41 \\
\hline Presence of GD-enhancement & 0.68 & 0.24 & 1.95 & 0.61 & 0.23 & 1.61 \\
\hline
\end{tabular}

*Based on 1000 bootstrap samples (bias corrected and accelerated)

${ }^{\#} p<0.05$ 
Table 4 Serious adverse events (SAEs) reported over 10 years of follow-up

\begin{tabular}{|c|c|c|c|c|c|}
\hline Escalation $(n=738)$ & & & Induction $(n=75)$ & & \\
\hline SAE name & Treatment taken at SAE & $n$ & & Treatment taken at SAE & \\
\hline Progressive multifocal leukoencephalopathy & $\begin{array}{l}\text { Natalizumab (pd) } \\
\text { Natalizumab (pd) } \\
\text { Natalizumab (pd) }\end{array}$ & 3 & $\begin{array}{l}\text { Solid malignancies } \\
\text { - Bowel } \\
\text { - Breast } \\
\text { - Lung } \\
\text { - Nasopharynx }\end{array}$ & $\begin{array}{l}\text { Interferon beta }(\mathrm{pd}) \\
\text { Interferon beta }(\mathrm{pd}) \\
\text { Interferon beta }(\mathrm{pd}) \\
\text { Natalizumab }(\mathrm{pd})\end{array}$ & \\
\hline $\begin{array}{l}\text { Solid malignancies } \\
\text { - Breast } \\
\text { - Uterus }\end{array}$ & $\begin{array}{l}\text { None (most recent: Mitoxantrone) } \\
\text { Natalizumab (pd) }\end{array}$ & 2 & Leukemia & $\begin{array}{l}\text { None (most recent: mitoxantrone) } \\
\text { None (most recent: mitoxantrone) }\end{array}$ & \\
\hline Pneumonia & Natalizumab & 2 & Febrile neutropenia & Mitoxantrone (pd) & 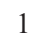 \\
\hline Myocardial infarction & $\begin{array}{l}\text { Interferon beta } \\
\text { Natalizumab }\end{array}$ & 2 & Myocardial infarction & Interferon beta & \\
\hline Brain aneurysm (accidental finding) & Interferon beta & 1 & & & \\
\hline Deep vein thrombosis & Interferon beta $(\mathrm{pd})$ & 1 & & & \\
\hline Grave's disease & None (most recent: alemtuzumab) & 1 & & & \\
\hline Hydronephrosis & Interferon Beta & 1 & & & \\
\hline Hyporegenerative anemia & Natalizumab (pd) & 1 & & & \\
\hline Leukemia & None (most recent: mitoxantrone) & 1 & & & \\
\hline Non-infectious hepatitis & Natalizumab (pd) & 1 & & & \\
\hline Psychosis & Interferon beta $(\mathrm{pd})$ & 1 & & & \\
\hline Status epilepticus & Azathioprine & 1 & & & \\
\hline
\end{tabular}

$p d$ : treatment permanently discontinued due to SAE

one can argue that not all patients may benefit from early immunosuppression to reset immune system over the longterm period.

Albeit encouraging, findings on early immunosuppression have raised relevant safety concerns; thus, their use has been restricted only to patients with aggressive MS [12]. In our study, induction was associated with an approximately fourfold increased risk of SAEs, especially malignant neoplasms, as compared to escalation. Treatment-related leukemia or lymphoma and infertility have been reported with both MTX and CYC [9, 10]. Specific side effects of dose-related cardiotoxicity and bladder malignancies have been associated with MTX and CYC, respectively [37, 38]. Consequently, administration of MTX and CYC has now been replaced by newer agents such as ALZ and CLB, in spite of some attempts for profiling patients who will have a more favorable risk:benefit profile [39]. Two pragmatic, randomized clinical trials are currently ongoing to elucidate if an early "aggressive" therapy approach, including induction with ALZ, will be associated with better outcome as compared with starting treatment with platform injective or oral drugs: the TRaditional versus Early Aggressive Therapy for MS (TREAT-MS, ClinicalTrials.gov no. NCT03500328) and the Determining the Effectiveness of Early Intensive Versus Escalation Approaches for the Treatment of RRMS (DELIVER-MS, ClinicalTrials.gov no. NCT03535298).
Our study is not without limitations, mainly due to its retrospective design, small sample size (especially for induction group), and comparison of patients in different treatment eras (MTX and CYC are not longer prescribed given the increased availability of newer drugs). Our data refer to specific treatments and are affected by the era in which they were collected; therefore, they cannot be generalized and extended to all current induction treatments. Although we are confident that our statistical approach, based on PS matching and pairwise comparisons $[24,40]$, allowed us to compare data of patients with similar baseline characteristics over a long-term follow-up, we cannot overcome the possibility of indication, selection, and hidden biases [41]. Indication bias refers to lack of randomization to treatment exposure [41]. Selection bias is mainly attributable to the exclusion from the analysis of a greater proportion of patients in the escalation than in the induction group as consequence of both the eligibility criteria and PSmatching procedure that is prone to the so-called bias due to incomplete matching [42]. This implies that the hypothesis regarding the superiority of induction versus escalation must be restricted only to older patients with higher EDSS scores (see also Table 1). Regarding hidden bias, we cannot exclude that induction was adopted in some patients due to unmeasured prognostic factors (not encompassed among the baseline variables), including (but not limited to) symptom onset, cognitive deficit, MRI characteristics including lesion burden, 
black holes, discernable brain atrophy, and infratentorial and/ or spinal cord involvement [12].

In conclusion, our study provides real-world evidence that initial treatment with an induction agent is associated with more favorable long-term benefits as compared to an escalation approach in patients with RRMS; this, at the price of an increased risk of SAEs and limitations in the future treatment sequencing. Moreover, our data suggest that the best candidate for induction is a younger patient in the early disease stage. Being based on observational data collected in the real-world setting and retrospectively analyzed, our findings should be considered only hypothesis-generating. We are also aware that immunosuppressant agents such as MTX and CYC are no longer in wide use for RRMS, but hopefully this study may represent an avenue for future investigation aimed to clarify if the newer induction agents, namely ALZ and CLB, could provide a more advantageous long-lasting risk:benefit profile.

\section{Compliance with Ethical Standards}

Required Author Forms Disclosure forms provided by the authors are available with the online version of this article.

Conflict of Interest The authors declare that they have no conflict of interest.

Financial Disclosures LP: consulting fees from Biogen, Novartis, and Roche; speaker honoraria from Biogen, Genzyme, Merck Serono, Novartis, and Teva; travel grants from Biogen, Genzyme, Novartis, and Teva; research grants from the Italian MS Society (Associazione Italiana Sclerosi Multipla) and Genzyme.

CRM: nothing to disclose.

VN: consulting fees from Novartis, Roche, Mylan, Biogen Idec, Merk Serono and Bayer Schering; speaker honoraria from Mylan, Teva, Biogen Idec, Sanofi Genzyme and Merk Serono; travel grants from Teva, Biogen Idec, Sanofi Genzyme, Roche and Novartis.

CMS: consulting fees for advisory board from Biogen, Merck Serono, Almirall, and GW Pharma; speaking honoraria from Biogen and Merck Serono; research grants and support by the FISM (Fondazione Italiana Sclerosi Multipla).

SH: fees as invited speakers or travel grants from Biogen, Novartis, Merck Serono, Roche, CSL Behrig, Bial.

$\mathrm{CC}$ : fees as invited speaker and travel grants for attending meeting from Serono, Biogen, Teva, Novartis.

LDG: travel grants from Biogen, Novartis, and Teva.

NDR: speaker honoraria from Biogen Idec, Genzyme, Novartis, Sanofi-Aventis; funding for participation in advisory board to Novartis and Genzyme-Sanofi and for travel to scientific meetings from Biogen Idec, Teva, Sanofi-Genzyme, Roche, Almirall, and Novartis.

SG: fees as invited speaker or travel expenses for attending meeting from Biogen, Merck-Serono, Teva, Almirall, Sanofi-Aventis, Novartis, and Genzyme.

SR: fees as invited speaker or travel expenses for attending meeting from Biogen, Merck-Serono, Teva, Sanofi, Novartis, Genzyme.

SR: speaking honoraria from Merck Serono and Teva.

CT: honoraria for speaking and travel grants from Biogen, SanofiAventis, Merck Serono, Bayer-Schering, Teva, Genzyme, Almirall, and Novartis.

RC: lecture fees and/or travel grants from Novartis, Biogen, Celgene, Novartis, TEVA, Genzyme, and Sanofi-Aventis.
MM: scientific advisory board membership of Bayer Schering, Biogen, Sanofi-Genzyme, Merck-Serono, Novartis, Teva; consulting and/or speaking fees, research support or travel grants from Almirall, Bayer Schering, Biogen, CSL Behring, Sanofi-Genzyme, Merck Serono, Novartis, Teva, Ultragenix; principal investigator in clinical trials for Biogen, Merck Serono, Novartis, Roche, Sanofi Genzyme, Teva, Ultragenix.

CG: fees as invited speaker or travel expenses for attending meeting from Biogen, Merck-Serono, Teva, Sanofi, Novartis, Genzyme.

Ethical Statement The Ethical Committee Board (Comitato Etico Lazio 1) provides exemption of approval for post-marketing, retrospective study not interfering in the standard of care received by patients. All data were gathered after an informed consent was obtained from each participant. This study was conducted in accordance with specific national laws and the ethics standards laid down in the 1964 Declaration of Helsinki and its later amendments. Anonymized data presented in this article will be made available at the request of a qualified investigator (requests should be sent to luca.prosperini@gmail.com).

\section{References}

1. Rotstein D, Montalban X. Reaching an evidence-based prognosis for personalized treatment of multiple sclerosis. Nat. Rev. Neurol. 2019:15(5):287-300.

2. Naismith RT. Multiple sclerosis therapeutic strategies: Start safe and effective, reassess early, and escalate if necessary. Neurol. Clin. Pract. 2011; 1(1):69-72.

3. Gasperini C, Prosperini L, Tintoré M, et al. Unraveling treatment response in multiple sclerosis: A clinical and MRI challenge. Neurology. 2019; 92(4):180-192.

4. Ransohoff RM, Hafler DA, Lucchinetti CF. Multiple sclerosis-a quiet revolution. Nat. Rev. Neurol. 2015; 11(3):134-142.

5. Ontaneda D, Tallantyre E, Kalincik T, et al. Early highly effective versus escalation treatment approaches in relapsing multiple sclerosis. Lancet. Neurol. 2019; 18(10):973-980.

6. Prosperini L, Kinkel RP, Miravalle AA, Iaffaldano P, Fantaccini S. Post-natalizumab disease reactivation in multiple sclerosis: systematic review and meta-analysis. Ther. Adv. Neurol. Disord. 2019; 12: 1756286419837809.

7. Giovannoni G. Disease-modifying treatments for early and advanced multiple sclerosis: a new treatment paradigm. Curr. Opin. Neurol.. 2018; 31(3):233-243.

8. Le Page E, Edan G. Induction or escalation therapy for patients with multiple sclerosis? Rev. Neurol. (Paris). 2018; 174(6):449-457.

9. Lebrun C, Rocher F. Cancer Risk in Patients with Multiple Sclerosis: Potential Impact of Disease-Modifying Drugs. CNS Drugs. 2018; 32(10):939-949.

10. Martinelli V, Cocco E, Capra R, et al. Acute myeloid leukemia in Italian patients with multiple sclerosis treated with mitoxantrone. Neurology. 2011; 77(21):1887-1895.

11. Grebenciucova E, Pruitt A. Infections in Patients Receiving Multiple Sclerosis Disease-Modifying Therapies. Curr. Neurol. Neurosci. Rep. 2017; 17(11):88.

12. Rush CA, MacLean HJ, Freedman MS. Aggressive multiple sclerosis: proposed definition and treatment algorithm. Nat. Rev. Neurol. 2015; 11(7):379-389.

13. Harding K, Williams O, Willis M, et al. Clinical Outcomes of Escalation vs Early Intensive Disease-Modifying Therapy in Patients With Multiple Sclerosis. JAMA Neurol. 2019;76(5):536541

14. Merkel B, Butzkueven H, Traboulsee AL, Havrdova E, Kalincik T. Timing of high-efficacy therapy in relapsing-remitting multiple 
sclerosis: A systematic review. Autoimmun. Rev. 2017; 16(6):658665.

15. Lublin FD, Reingold SC, Cohen JA, et al. Defining the clinical course of multiple sclerosis: the 2013 revisions. Neurology. 2014; 83(3):278-286.

16. Fisniku LK, Brex PA, Altmann DR, et al. Disability and T2 MRI lesions: a 20-year follow-up of patients with relapse onset of multiple sclerosis. Brain 2008; 131(Pt 3):808-817.

17. Degenhardt A, Ramagopalan SV, Scalfari A, Ebers GC. Clinical prognostic factors in multiple sclerosis: a natural history review. Nat. Rev. Neurol. 2009; 5(12):672-682.

18. Brown JWL, Coles A, Horakova D, et al. Association of Initial Disease-Modifying Therapy With Later Conversion to Secondary Progressive Multiple Sclerosis. JAMA. 2019; 321(2):175-187.

19. Kurtzke JF. Rating neurologic impairment in multiple sclerosis: an expanded disability status scale (EDSS). Neurology. 1983; 33(11): 1444-1452.

20. Confavreux C, Vukusic S, Adeleine P. Early clinical predictors and progression of irreversible disability in multiple sclerosis: an amnesic process. Brain 2003; 126(Pt 4):770-782.

21. Le Page E, Leray E, Taurin G, et al. Mitoxantrone as induction treatment in aggressive relapsing remitting multiple sclerosis: treatment response factors in a 5 year follow-up observational study of 100 consecutive patients. J. Neurol. Neurosurg. Psychiatry. 2008; 79(1):52-56.

22. Río J, Nos C, Tintoré M, et al. Defining the response to interferonbeta in relapsing-remitting multiple sclerosis patients. Ann. Neurol. 2006; 59(2):344-352.

23. Austin PC. Balance diagnostics for comparing the distribution of baseline covariates between treatment groups in propensity-score matched samples. Stat. Med. 2009; 28(25):3083-3107.

24. Cummings P, McKnight B, Greenland S. Matched cohort methods for injury research. Epidemiol. Rev. 2003; 25:43-50.

25. Péron J, Buyse M, Ozenne B, Roche L, Roy P. An extension of generalized pairwise comparisons for prioritized outcomes in the presence of censoring. Stat. Methods Med. Res. 2018; 27(4):1230 1239.

26. Rassen JA, Shelat AA, Myers J, et al. One-to-many propensity score matching in cohort studies. Pharmacoepidemiol. Drug Saf. 2012; 21 Suppl 2:69-80.

27. Confavreux $\mathrm{C}$, Vukusic $\mathrm{S}$. The clinical course of multiple sclerosis. Handb. Clin. Neurol. 2014; 122:343-369.

28. Pardo G, Jones DE. The sequence of disease-modifying therapies in relapsing multiple sclerosis: safety and immunologic considerations. J. Neurol. 2017; 264(12):2351-2374.

29. Döhner H, Estey EH, Amadori S, et al. Diagnosis and management of acute myeloid leukemia in adults: recommendations from an international expert panel, on behalf of the European LeukemiaNet. Blood. 2010; 115(3):453-474.

30. Verhoeven MMA, Welsing PMJ, Bijlsma JWJ, et al. Effectiveness of Remission Induction Strategies for Early Rheumatoid Arthritis: a
Systematic Literature Review. Curr. Rheumatol. Rep. 2019; 21(6): 24. Available at: https://www.ncbi.nlm.nih.gov/pmc/articles/ PMC6478774/ [Accessed July 26, 2019].

31. Vollmer T, Panitch H, Bar-Or A, et al. Glatiramer acetate after induction therapy with mitoxantrone in relapsing multiple sclerosis. Mult. Scler. 2008; 14(5):663-670.

32. Edan G, Comi G, Le Page E, et al. Mitoxantrone prior to interferon beta-1b in aggressive relapsing multiple sclerosis: a 3-year randomised trial. J. Neurol. Neurosurg. Psychiatry. 2011; 82(12): $1344-1350$

33. Harrison DM, Gladstone DE, Hammond E, et al. Treatment of relapsing-remitting multiple sclerosis with high-dose cyclophosphamide induction followed by glatiramer acetate maintenance. Mult. Scler. 2012; 18(2):202-209.

34. CAMMS223 Trial Investigators, Coles AJ, Compston DAS, et al. Alemtuzumab vs. interferon beta-1a in early multiple sclerosis. N. Engl. J. Med. 2008; 359(17):1786-1801.

35. Cohen JA, Coles AJ, Arnold DL, et al. Alemtuzumab versus interferon beta $1 \mathrm{a}$ as first-line treatment for patients with relapsingremitting multiple sclerosis: a randomised controlled phase 3 trial. Lancet 2012; 380(9856): 1819-1828.

36. Leist TP, Comi G, Cree BAC, et al. Effect of oral cladribine on time to conversion to clinically definite multiple sclerosis in patients with a first demyelinating event (ORACLE MS): a phase 3 randomised trial. Lancet Neurol. 2014; 13(3):257-267.

37. Marriott JJ, Miyasaki JM, Gronseth G, O'Connor PW, Therapeutics and Technology Assessment Subcommittee of the American Academy of Neurology. Evidence Report: The efficacy and safety of mitoxantrone (Novantrone) in the treatment of multiple sclerosis: Report of the Therapeutics and Technology Assessment Subcommittee of the American Academy of Neurology. Neurology. 2010; 74(18):1463-1470.

38. De Ridder D, van Poppel H, Demonty L, et al. Bladder cancer in patients with multiple sclerosis treated with cyclophosphamide. $J$. Urol. 1998; 159(6):1881-1884.

39. Hasan SK, Buttari F, Ottone T, et al. Risk of acute promyelocytic leukemia in multiple sclerosis: coding variants of DNA repair genes. Neurology. 2011; 76(12):1059-1065.

40. Trojano M, Pellegrini F, Paolicelli D, Fuiani A, Di Renzo V. Observational studies: propensity score analysis of non-randomized data. Int. MS J. 2009; 16(3):90-97.

41. Kalincik T, Sormani MP. Reporting treatment outcomes in observational data: A fine balance. Mult. Scler. 2017; 23(1):21-22.

42. Austin PC. Double propensity-score adjustment: A solution to design bias or bias due to incomplete matching. Stat Methods Med. Res.. 2017; 26(1):201-222.

Publisher's Note Springer Nature remains neutral with regard to jurisdictional claims in published maps and institutional affiliations.

\section{Affiliations}

\section{Luca Prosperini ${ }^{1}$ - Chiara Rosa Mancinelli ${ }^{2}$. Claudio Marcello Solaro ${ }^{3}$ - Viviana Nociti ${ }^{4,5}$. Shalom Haggiag ${ }^{1}$. Cinzia Cordioli ${ }^{2}$ - Laura De Giglio ${ }^{6,7}$ - Nicola De Rossi ${ }^{2}$ - Simonetta Galgani ${ }^{1}$ - Sarah Rasia ${ }^{2}$. Serena Ruggieri ${ }^{1,7}$. Carla Tortorella $^{1} \cdot$ Ruggero Capra $^{2} \cdot$ Massimiliano Mirabella $^{4,5} \cdot$ Claudio Gasperini $^{1}$}

1 Department of Neurosciences, S. Camillo-Forlanini Hospital, C.ne Gianicolense 87, 00152 Rome, Italy
2 Multiple Sclerosis Centre, ASST Spedali Civili di Brescia, P.O. Montichiari, Via Ciotti 154, 25018 Montichiari, BS, Italy 
3 Rehabilitation Unit 'Mons. L. Novarese' Hospital, Loc. Trompone, 13040 Moncrivello, VC, Italy

4 Fondazione Policlinico Universitario 'A. Gemelli' IRCCS, L.go A. Gemelli 8, 00168 Rome, Italy

5 Università Cattolica del Sacro Cuore, L.go A. Gemelli 8, 00168 Rome, Italy
6 Unit, S. Filippo Neri Hospital, Via G. Martinotti 20, 00135 Rome, Italy

7 Multiple Sclerosis Centre, S. Andrea Hospital, Via di Grottarossa 1035, 00189 Rome, Italy 